\title{
Motivación de maestros de infantil y primaria durante la formación inicial universitaria
}

\section{Motivation of childhood and primary teachers during initial university education}

\author{
Vicente Llorent-Bedmar \\ Universidad de Sevilla (España) \\ llorent@us.es \\ Verónica Carmen Cobano-Delgado \\ Universidad de Sevilla (España) \\ cobano@us.es \\ Purificación Bejarano-Prats \\ Universidad de Córdoba (España) \\ p.bejarano@magisteriosc.es \\ Fecha de recepción: 12/01/2018 \\ Fecha de aceptación: 20/08/2019
}

\section{Resumen}

La importancia de la motivación del alumnado de Magisterio es clave para cualquier sistema educativo. Para mejorarla realizamos un análisis de determinadas características personales del alumnado que cursa los Grados de Educación Infantil y Primaria en Andalucía y las relacionamos con su nivel y tipo de motivación (intrínseca y extrínseca). Utilizamos un diseño mixto; realizamos investigación documental, diseñamos un cuestionario ad hoc y la entrevista. Concretamente se han analizado 996 cuestionarios y 56 entrevistas. Los constructos del cuestionario se validaron mediante Análisis Factorial Confirmatorio, y para el análisis bivariante utilizamos las pruebas paramétricas y no paramétricas correspondientes. Entre los resultados, constatamos diferencias significativas entre los tipos de motivación y determinadas variables de índole académica, familiar y socio-económica. Concluimos la importancia de favorecer la motivación intrínseca de los futuros maestros aplicando programas adecuados de orientación vocacional, realizando una elección de titulación personal y razonada, así como implicándose en labores de voluntariado.

Palabras clave: Docente; Motivación; Alumnado universitario; Formación; Andalucía (España) 


\begin{abstract}
The motivation of Teacher Training students is key for any educational system. In order to improve their motivation we have carried out a study of specific personal characteristics of the students of the degrees in Childhood and Primary Education in Andalusia and we have related it to their level and type of motivation (intrinsic and extrinsic). We use a mixed design; we carried out a documentary research, we designed a questionnaire ad hoc and an interview. Specifically 996 questionnaires were analyzed and 56 interviews. The constructs of the questionnaire have been validated using Confirmatory Factor Analysis, and for bivariate analysis we have carried out the corresponding parametric and non-parametric tests. Among the main results, we have identified significant differences between the types of motivation and certain academic, family and socio-economic variables. We concluded the importance of favoring intrinsic motivation in future teachers by applying appropriate programmes of vocational guidance, to make a choice with regard to the qualification on a personal and reasoned level as well as the involvement in volunteer activities.
\end{abstract}

Keywords: Teacher; Motivation; University students; Training; Andalusia (Spain)

\title{
1. INTRODUCGIÓN
}

La motivación incide en la elección realizada sobre determinadas acciones personales, y repercute en la persistencia y el esfuerzo dedicado a llevar a cabo dichas tareas. Dörnyei y Ushioda (2013) indican que la motivación permite reconocer los motivos por los cuales las personas deciden realizar alguna actividad, así como el tiempo y el esfuerzo que están dispuestos a emplear para su consecución.

Definimos la motivación como la energía que impulsa a una determinada acción (Bhuiyan et al., 2015), siendo un elemento que condiciona la vida de la persona (Siwek, Oleszkowicz y Słowińska, 2016).

En el ámbito educativo, son muchos los autores que señalan la relación entre motivación y aprendizaje (entre otros, Elias y Sánchez-Gelabert, 2014; Presentación et $a l ., 2015)$. La motivación es fundamental en el proceso de enseñanza-aprendizaje y le otorga sentido y significado al conocimiento adquirido (Reyes, 2015). Concretamente, Del Siegle et al. (2014) subrayan la importancia de la motivación académica para la persistencia del alumnado en las tareas, en su rendimiento académico y en la elección de la carrera.

Además, las consecuencias de la motivación varían en función de su modalidad, intrínseca o extrínseca, así como en función de las estrategias de aprendizaje (Ahn y Shin, 2014). Son diversas las investigaciones que contemplan distintos tipos de motivación, tales como las realizadas por Bailey y Phillips (2016); Hidi (2015); Niehaus et al. (2012); Pan et al. (2013) y Valle et al. (2015). Por un lado, la motivación intrínseca permite al alumnado estar más dispuesto a realizar un importante esfuerzo mental, y a utilizar estrategias de aprendizaje más profundas 
Revista de Humanidades, 38 (2019). p.37-64. ISSN 1130-5029

y efectivas realizando la tarea por el interés y curiosidad que la misma le genera. Mientras que, por otro lado, el alumnado motivado extrínsecamente habitualmente selecciona aquellas tareas que les resultan más fáciles y con las que pueden asegurarse obtener recompensas externas (Henaín, 2015; Rinaudo et al., 2003).

En este sentido, considerando la importancia de la motivación intrínseca nos cuestionamos cómo podemos mejorarla en el alumnado que cursa los Grados de Educación Infantil y Educación Primaria. Así, se diferenciaron motivos de carácter intrínseco y extrínseco por los cuales eligieron dichas titulaciones. Además, se estimó relevante conocer determinadas características, factores personales que pudieran estar relacionados con su motivación, con la intención de identificar estrategias motivacionales más eficaces.

\section{FAGTORES QUE INCIDEN EN EL NIVEL DE MOTIVACIÓN DEL ALUMNADO}

Se pueden señalar algunos aspectos que repercuten en la motivación, la investigación de Meijers et al. (2013) expone que el conocimiento sobre las actitudes y deseos del alumnado contribuyen a su motivación por aprender. Estudios como el de Cabanach, et al. (2010) ponen de manifiesto que la creencia positiva de cada discente sobre su autoeficacia, además de un factor de protección ante el estrés, mejora su motivación. Espinar y Ortega (2015) destacan la importancia de la figura del profesor entre los factores que afectan a la motivación del alumnado. En esta misma línea, puntualizan De Boer et al. (2010) que también afectan decisivamente a la motivación las expectativas del profesorado.

En este sentido, destacamos la importancia de la figura del docente para cualquier sistema escolar. "El Informe McKinsey, a partir de la comparación de los 10 mejores sistemas educativos del mundo (...), destaca que el nivel educativo de un país depende de la formación, motivación y aprendizaje permanente de sus profesores y profesoras" (Consejería de Educación. Junta de Andalucía, 2016, p. 33).

Reseñamos que la investigación realizada por De Cooman_et al. (2007) ya concluía que el profesorado recién titulado que ejerce la profesión considera los aspectos de carácter intrínseco, altruista e interpersonal como relevantes para su desempeño profesional.

El docente debe estar suficientemente motivado para desarrollar un estilo de actuación que facilite el aprendizaje del alumnado. Debe enseñar a utilizar habilidades motivadoras que ayuden a los discentes a aprender (González-Peiteado, 2013). Sin embargo, según exponen Sarsenbaykyzy et al. (2016) hay alumnado de Magisterio que pierde la motivación al dudar si tienen determinadas cualidades para el ejercicio de la profesión, tales como habilidades profesionales, desarrollo intelectual y creatividad. 
Este aspecto es de interés, concretamente, identificamos como factores desmotivadores, que el alumnado pueda presentar un comportamiento de indefensión, considerando que su éxito académico es debido a razones externas que no puede controlar. Son personas que consideran tener escasa habilidad (poca inteligencia, falta de memoria...) y sienten aburrimiento o ansiedad en el desempeño de sus tareas (Naranjo, 2009). Asimismo, puede manifestar desesperanza aprendida el alumnado que piensa que está destinado a fracasar, independientemente de sus logros académicos. En este sentido, Ilustrisimo (2016) indica que tener una perspectiva fatalista sobre el futuro actúa como indicador de la desmotivación.

Además de diferentes estudios e investigaciones que analizan aspectos que inciden en la motivación, indagamos a continuación sobre la relevancia que esta tiene en la Universidad. Resulta necesario concienciar de la importancia de la motivación intrínseca en el ámbito universitario, concretamente en las titulaciones de Magisterio, aspecto crucial para el buen desempeño de una óptima labor profesional docente.

\section{MOTIVACIÓN EN LOS ESTUDIOS UNIVERSITARIOS}

La motivación tiene un papel preponderante en el aprendizaje universitario, como se infiere en recientes investigaciones realizadas por Dryer et al. (2016) y García-Ramírez (2016). Este último concluye que en la innovación docente, la motivación (por competencias y de logro) es clave para el desarrollo del pensamiento creativo y para mejorar el rendimiento académico.

La investigación realizada por Donche et al. (2013) analiza la personalidad, motivación académica y las estrategias de aprendizaje del alumnado en el primer año de universidad. Asimismo, Liu et al. (2014) estudiaron los perfiles de motivación, las estrategias de aprendizaje y el rendimiento académico; y determinaron con los resultados obtenidos del análisis multivariante de la varianza la importancia de la satisfacción de las necesidades para un aprendizaje autorregulado.

Por tanto, como ya indicamos, en la formación inicial adquiere importancia promover y despertar en los universitarios una fuerte motivación para la adquisición de nuevos aprendizajes y el desarrollo de competencias, facilitándoles así el gusto por continuar formándose e ir mejorando durante su desempeño profesional como docentes (Rosales, 2014).

Así, resulta necesaria en la sociedad actual una formación inicial exigente y esmerada en la mejora de la motivación intrínseca de todo el alumnado que ha accedido a los Grados de Educación Infantil y Educación Primaria, favoreciendo que cada universitario encuentre el verdadero sentido por el que desarrolla su profesión.

En esta línea, indicar que el alumnado que durante la formación inicial en la universidad empieza a tener interés profesional, consigue con mayor rapidez y efectividad su inserción en el mundo laboral; sin embargo, los que tienen una 
Revista de Humanidades, 38 (2019). p.37-64. ISSN 1130-5029

motivación extrínseca les resulta más factible que abandonen su profesión en sus primeros años de ejercicio (García, 2011).

También debemos señalar causas que ocasionan desmotivación, resultando este un problema que puede llegar a originar el abandono de los estudios universitarios (Bailey y Phillips, 2016; Ilustrisimo, 2016). Los motivos que generan en el alumnado la decisión de abandonar la formación universitaria han sido también estudiados por autores como Boza y Toscano (2012), quienes, en primer lugar, detectan la importancia de la escasa vocación y, en segundo lugar, señalan la baja motivación. Añaden que la motivación intrínseca ejerce mayor influencia que la extrínseca en este tipo de decisiones de renuncia.

Investigaciones como la de Aliakbari y Hemmatizad (2015) amplían los factores que influyen en la desmotivación. En este caso, refiriéndose al alumnado iraní de educación secundaria y universitaria, e identifican los siguientes factores desmotivacionales: el contenido y materiales de aprendizaje, los profesores y sus estilos de enseñanza, las instalaciones escolares, la falta de motivación intrínseca y los malos resultados académicos.

Concretamente en Andalucía, lugar donde se circunscribe esta investigación, se establece en el Plan de Éxito Educativo 2016-2020 como una línea de mejora la formación del profesorado. Entre otras actuaciones especifica "un modelo de formación inicial del profesorado más ajustado a las necesidades reales del sistema" (Consejería de Educación. Junta de Andalucía, 2016, p. 5). Por tanto, resulta del todo relevante realizar investigaciones que mejoren la formación inicial de futuros docentes y fomenten su motivación intrínseca.

De esta forma, este estudio aporta el análisis de determinadas características del alumnado que cursa los Grados de Educación Infantil y Primaria en Andalucía y su posible relación con su motivación. Concretamente se presentan factores de tipo personal, económico y familiar, que pueden relacionarse con la motivación intrínseca y extrínseca de los futuros maestros ${ }^{1}$. Aspectos que deben ser reconocidos por los docentes universitarios para el mejor desempeño de su labor profesional y en el diseño y desarrollo de programas de orientación profesional.

\section{OBJETIVOS Y METODOLOGÍA}

Fundamentada la relevancia de la motivación intrínseca en el alumnado de Magisterio, nos proponemos concienciar de la importancia de fomentarla y establecer propuestas para su mejora. Para ello, planteamos los siguientes objetivos específicos:

1 Este artículo se enmarca dentro de la Tesis Doctoral titulada Motivación de los futuros maestros en las universidades andaluzas. Estudio comparado del alumnado que cursa los Grados de Educación Infantil y Primaria. Dirigida por Vicente Llorent Bedmar y Verónica Cobano-Delgado Palma. Realizada por Purificación Bejarano Prats. Universidad de Sevilla, Sevilla. 
Motivación de maestros de infantil y primaria... - V. Llorent-Bedmar y otros

- Objetivo 1:

Definir y estudiar la motivación intrínseca y extrínseca del alumnado de Magisterio de Educación Infantil y Primaria en Andalucía.

- Objetivo 2:

Analizar factores de índole académica, familiar y socio-económica del alumnado que cursa los Grados de Educación Infantil y Educación Primaria en Andalucía.

- Objetivo 3:

Conocer la relación de determinados factores académicos, familiares y socioeconómicos del alumnado de Magisterio en Andalucía con el nivel y tipo de motivación (intrínseca o extrínseca).

También formulamos las hipótesis, que fueron determinadas cuando ya se tenía conocimiento sobre la temática. De manera general planteamos que la motivación intrínseca durante la formación inicial en las titulaciones de Magisterio es fundamental para el proceso de aprendizaje y el desempeño de su futura labor profesional. De forma más específica detallamos que: (1) el conocimiento de las características personales del alumnado de Magisterio permite el planteamiento de estrategias más eficaces para la mejora de su motivación intrínseca, (2) el momento de elección de la titulación de Magisterio puede tener relación con el nivel de motivación del alumnado y (3) determinados factores relacionados con el contexto del alumnado (familiar y socio-económico) se pueden relacionar con su motivación por la titulación de Magisterio.

Pues bien, para llevar a cabo este estudio seguimos los principios éticos que desde la investigación educativa se deben seguir (Sañuedo, 2006). Planteamos un diseño mixto, combinando técnicas cuantitativas y cualitativas para tener una mejor comprensión del tema de estudio. Desde este planteamiento, como indica Pereira (2011) "no solo se desea la obtención de datos numéricos, sino también se busca la visión más íntima del participante, los datos cualitativos cobran un papel relevante" (p. 26).

\subsection{Participantes}

La población está constituida por el alumnado de primero y cuarto curso de los Grados de Educación Infantil y Primaria de todas las facultades de educación de Andalucía (España), tanto en los centros públicos como en los privados que están adscritos a una universidad. Es decir, los centros de Almería, Cádiz, Córdoba, Granada, Huelva, Jaén, Málaga, Sevilla, Antequera, Bormujos, Granada, Córdoba, La Línea de la Concepción y Úbeda. 
Revista de Humanidades, 38 (2019). p.37-64. ISSN 1130-5029

La muestra obtenida tiene en cuenta aspectos como el nivel de fiabilidad y grado de precisión, exigibles a la hora de hacer inferencias con los datos de la muestra elegida. Utilizamos la fórmula para el caso de estimación de proporciones para poblaciones finitas y calculamos estableciendo el tamaño de la población $\mathrm{N}=$ 14 931; $Z_{1-\alpha / 2}=1,96$; el valor de la distribución normal tipificada dado por el nivel de confianza exigido del 95\% (nivel de significación $\alpha=0,05$ ) y un margen de error para la proporción muestral (error muestral) e $=0,03$ (Albert, 2007). Consideramos p.q $=0,25$ con el propósito de obtener un tamaño de la muestra (n) que asegure una representatividad general, donde $\mathrm{p}$ es la proporción poblacional que presenta una variable de estudio y q su complementaria, $\mathrm{q}=1-\mathrm{p}$ (Vivanco, 2005). Como resultado obtenemos un tamaño de la muestra de $\mathrm{n}=996$ discentes (n) a los cuales se les ha aplicado el cuestionario diseñado.

En el caso de las entrevistas, aplicamos un total de 56. Matizamos que, habiendo obtenido el principio de saturación teórica, según los criterios de muestreo cualitativo cuando los datos resultan repetitivos (Valles, 2014), continuamos realizando entrevistas para obtener información del alumnado de los dos cursos (primero y cuarto) de ambas titulaciones (Infantil y Primaria) y de los distintos centros investigados.

\subsection{Instrumentos}

Se ha diseñado un cuestionario ad hoc para la tesis doctoral titulada Motivación de los futuros maestros en las universidades andaluzas. Estudio comparado del alumnado que cursa los Grados de Educación Infantil y Primaria, ha sido el principal instrumento de recogida de información utilizado, cumple con los requisitos de validez y fiabilidad (Padilla, 2002). Esta tarea no ha sido fácil, debido a la dificultad que supone la medición de fenómenos como la motivación. Utilizamos un procedimiento de análisis que permite el estudio de "variables latentes inobservables por medio de indicadores" (Bisquerra, 2000, p. 89).

Dicho cuestionario consta de cuatro apartados, en este artículo profundizamos en dos de ellos: (1) los datos personales del alumnado que cursa los Grados de Educación Infantil y Primaria en Andalucía y (2) el referido a los motivos iniciales para la elección del Grado. Este último bloque se desarrolla en base a la bibliografía consultada, entre otros autores Naranjo (2009) detalla los tipos de motivación intrínseca y extrínseca, Printrich y DeGroot (citado en Rodríguez, 2009) plantea la importancia del componente de valor - motivos por los que se realiza una tarea- y Bertomeu et al. (2007) investiga sobre las motivaciones hacia los estudios de Magisterio.

La validación de constructos (las motivaciones intrínseca y extrínseca) del cuestionario diseñado, realizada mediante Análisis Factorial Confirmatorio (AFC), que "se centra en el estudio de los modelos de medida, esto es, en analizar las relaciones entre un conjunto de indicadores o variables observadas y una o más 
variables latentes o factores" (Arias, 2008, p. 75). En este caso, los indicadores han sido los ítems del cuestionario.

Para medir los tipos de motivación intrínseca y extrínseca hemos agrupado determinadosítems del cuestionario. Realizamos el cuestionariopilotoy analizamos los resultadosobtenidos, observamoslanecesidaddereformularciertosítemsparaaumentar la consistencia interna y validar posteriormente la teoría mediante el AFC. Podemos observar en la Tabla 1 los valores de Alpha de Cronbach de los constructos estudiados.

Tabla 1. Agrupación teórica de constructos y valores Alpha de Cronbach

\begin{tabular}{cccc} 
& Variables & Alpha de Cronbach (Piloto) & Alpha de Cronbach (Total) \\
\hline \multirow{2}{*}{ Motivación } & Intrínseca & 0,666 & 0,763 \\
& Extrínseca & 0,441 & 0,705 \\
\hline
\end{tabular}

Fuente. Elaboración propia.

Tras el análisis, finalmente identificamos seis ítems para medir el tipo de motivación intrínseca y once para la extrínseca. A continuación, la Tabla 2 muestra para los dos constructos, tanto para la motivación intrínseca como para la extrínseca, los ítems del cuestionario analizados.

Para realizar el AFC se utiliza el paquete de R Lavaan (software libre). Ajustamos los diferentes modelos de motivación (extrínsica e intrínseca) utilizando los ítems teóricos dentro de cada dimensión definida (Rosseel, 2015).

Comprobamos la estructura conceptual del instrumento para precisar grupos de indicadores o factores cuyos componentes medibles tratan un tema común (inter-correlaciones entre las respuestas a las cuestiones) definiendo a continuación el constructo como predice la teoría y eliminando las variables que no muestran correlación entre sí (Lei y Wu, 2007).

Los modelos de ecuaciones estructurales conocidos como SEM tienen la ventaja de "proponer el tipo y dirección de las relaciones que se espera encontrar entre las diversas variables contenidas en él, para pasar posteriormente a estimar los parámetros que vienen especificados por las relaciones propuestas a nivel teórico" (Ruiz et al., 2010, p. 34).

A continuación, presentamos los dos constructos (motivación intrínseca y extrínseca) formados por los ítems correspondientes. Observamos en la Figura 1 los índices de ajuste relativo, Comparative Fit Index (CFI) y Tucker Lewis Index (TLI), que muestran el buen ajuste de los datos a las escalas teóricas planteadas. Los valores de ambos ítems varían entre 0 y 1 , indicando 0 un mal ajuste y 1 un ajuste perfecto (Herrero, 2010). Consideramos el valor apropiado a partir de 0,9 (Hu y Bentler, 1999). También indicamos los resultados de los índices de ajuste absoluto, 
Revista de Humanidades, 38 (2019). p.37-64. ISSN 1130-5029

Tabla 2. Definición de constructos. Motivación intrínseca y extrínseca

\begin{tabular}{llc} 
Constructo & Denominación del ítem & No de ítem \\
\hline & Me gustaba la enseñanza & 1 \\
& Me gustaba trabajar con los niños & 2 \\
Motivación & Deseaba aprender temas educativos & 7 \\
& Pensaba que era gratificante educar a niños pequeños & 9 \\
& Deseaba ayudar a los demás & 16 \\
\hline & Tenía vocación & 3 \\
& Pensaba que era una titulación fácil & 4 \\
& Creía que era una profesión con buen salario & 5 \\
& Titulación bien considerada & 8 \\
Motivación & Un páa que era una profesión que tiene muchas vacaciones & 10 \\
extrínseca & Por tradición familiar & 11 \\
& Por imposición familiar & 12
\end{tabular}

Fuente. Elaboración propia.

Root Mean Square Error of Approximation (RMSEA) y su intervalo de confianza del 90\%, así como el valor de Standardized Root Mean Residual (SRMR). Un buen ajuste de los datos requiere que el intervalo superior para la medida de RMSEA y el valor de SRMR sea inferior a 0,1; variación que depende del autor referido y del tipo de modelo (Hu y Bentler, 1999; Hopper et al., 2008).

Asimismo, observamos en la Figura 1 los valores de los parámetros estandarizados entre las relaciones de los ítems con sus constructos y las asociaciones entre los constructos, junto con una indicación de la probabilidad (p-valor) asociada al parámetro. Los indicadores expresan el buen ajuste de los datos a la estructura teórica planteada, por tanto, obtenemos la validación de dichas escalas en sus correspondientes constructos.

Podemos identificar en la Figura 1 que los ítems referidos a la motivación intrínseca muestran cargas positivas, es decir, una mayor valoración en estos indicadores implica una mayor motivación intrínseca, siendo más representativa en las preguntas $2(0,712)$ y $16(0,703)$, y alcanza escasa representatividad en los ítems $9(0,466)$ y $6(0,499)^{2}$. En el caso de la motivación extrínseca, los 11 ítems expresan cargas positivas, siendo más representativa en la variable $4(0,686)$ y menos en la 15

2 En la Tabla 1 podemos identificar las variables correspondientes a cada ítem. 
$(0,227)^{3}$. También conviene reseñar la bidireccionalidad en la motivación intrínseca y extrínseca, resultando una relación negativa, es decir, cuando una aumenta la otra disminuye.

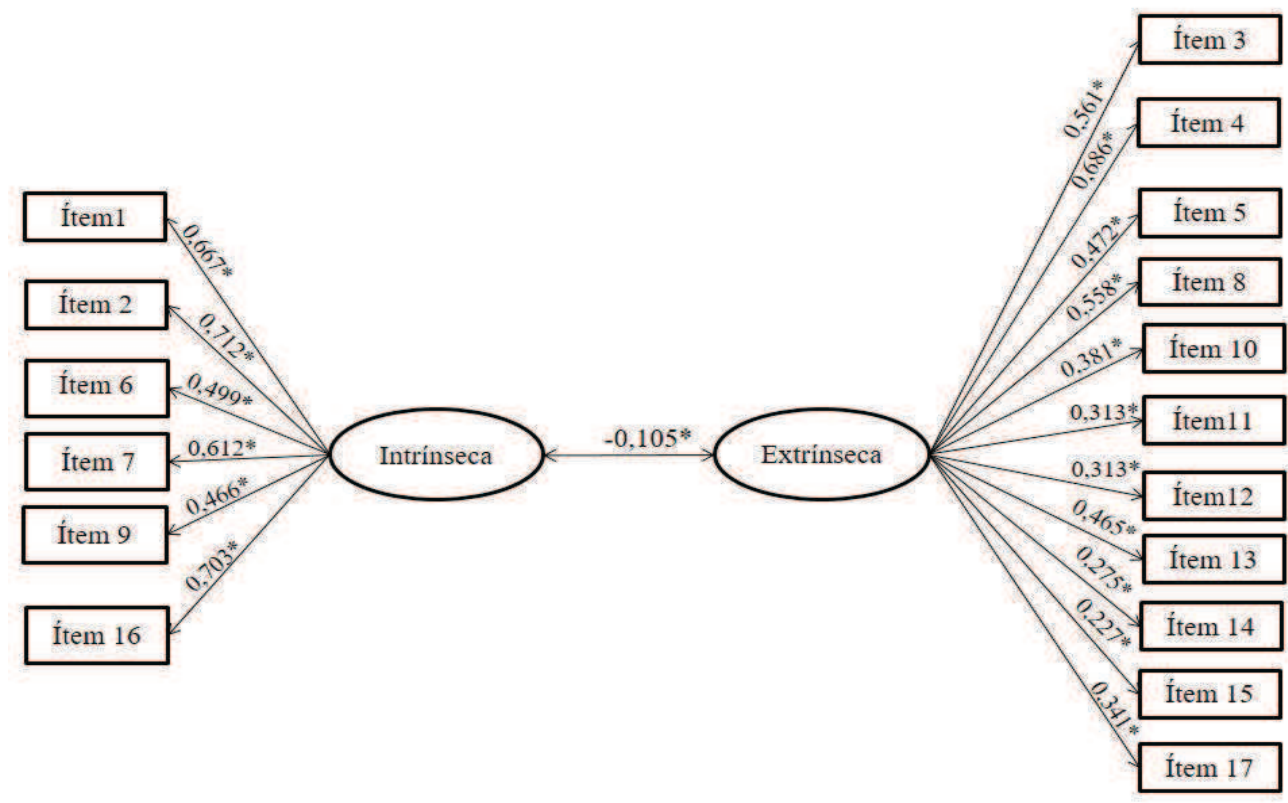

Figura 1. Constructos sobre la motivación (instrínseca y extrínseca). Método MLR; CFI=0,9; TLI=0,9; RMSEA=0,09 (0,085,0,095); $\mathrm{SRMR}=0,08: * \mathrm{p}<0,005$; $* * p<0,05$

Fuente: Elaboración propia.

Además del cuestionario y la investigación documental, las entrevistas personales al alumnado de los Grados de Infantil y Primaria nos han permitido profundizar en determinados aspectos del estudio. Se procedió a realizar la cédula de la entrevista, ya realizado el análisis documental, diseñado el cuestionario y analizados los datos de la prueba piloto del mismo. La relación interpersonal de la entrevista resultó de gran interés, ya que, a diferencia del cuestionario, permitió "la posibilidad de observar al sujeto y la forma en que se enfrenta a la situación" (Padilla, 2002, p. 142). En este caso, el procedimiento de análisis se ha efectuado con el programa informático MAXQDA, versión 11 para Windows. Para utilizar este programa informático se categorizaron dimensiones para el análisis, a las cuales se les asignó un color, agrupando así diferentes variables de la entrevista. En este artículo se aportan los datos de la dimensión referida a "motivos para continuar los estudios".

3 Ídem. 
Revista de Humanidades, 38 (2019). p.37-64. ISSN 1130-5029

\subsection{Procedimiento}

Para esta investigación, concluida durante el curso 2014-15, utilizamos pruebas paramétricas y no paramétricas para el análisis bivariante. Aplicamos pruebas paramétricas Analysis Of Variance (ANOVA) y la t de Student (t), las cuales comparan las medias aritméticas de los valores de la variable dependiente (McMillan y Schumacher, 2005).

La prueba ANOVA (F) la hemos utilizado cuando la variable independiente era politómica (más de dos modalidades) y con muestras (grupos muestrales) no relacionadas, es decir, excluyentes entre sí (cada sujeto presenta una sola modalidad de la variable independiente). También, hemos realizado la prueba de Welch (W). La prueba t de Student (t) la aplicamos con variables independientes dicotómicas (con solo dos modalidades) (Field, 2009).

En todas las pruebas de contraste de hipótesis el nivel de significación prefijado $\alpha=0,05$. De tal forma que si la probabilidad (p) asociada al valor numérico del estadístico de la prueba es menor o igual a 0,05 , consideramos diferencias significativas entre medidas, o distribuciones, de la variable dependiente observadas en grupos muestrales constituidos según cada modalidad de la variable independiente; por lo que podríamos concluir que las variables están relacionadas entre sí (Pérez et al., 2012).

Por último, señalar que hemos obtenido los resultados estadísticos del análisis bivariante empleando el software SPSS versión 21.

\section{RESULTADOS}

Con el análisis documental realizado en diversidad de estudios e investigaciones y desde el convencimiento de que el ambiente condiciona a la persona, estimamos que el tipo de motivación podría estar condicionado por tres tipos de factores: los académicos, los familiares y los socio-económicos. Teniendo en cuenta los tipos de motivación, intrínseca y extrínseca, del alumnado de los Grados de Infantil y Primaria, exponemos a continuación los resultados obtenidos según los tres factores indicados.

\subsection{Características académicas}

Para concretar las características académicas de los universitarios estudiamos las siguientes variables: a) estudios cursados antes de iniciar los Grados en Infantil o en Primaria ${ }^{4}$ (entre las opciones: Bachillerato ${ }^{5}$, Ciclo Formativo de Grado

4 Artículo 3 del Real Decreto 412/2014, de 6 de junio, por el que se establece la normativa básica de los procedimientos de admisión a las enseñanzas universitarias oficiales de Grado.

5 En España, el Bachillerato forma parte de la educación secundaria postobligatoria, y por lo tanto tiene carácter voluntario. Comprende dos cursos académicos, que se realizan ordinariamente entre los 16 y 18 años de edad (Ministerio de Educación, Cultura y Deporte, 2016a). 
Superior ${ }^{6}$, otros estudios universitarios), b) momento de elección para realizar los estudios universitarios de Magisterio (desde siempre, al terminar la Educación Secundaria Obligatoria ${ }^{7}$, al terminar Bachillerato, al terminar la Prueba de Acceso a la Universidad ${ }^{8}$, al terminar Ciclo Formativo de Grado Superior o en otro momento) y c) la opción en la que han elegido esta titulación (primera, segunda, tercera, cuarta o más).

Pues bien, en la primera variable hallamos diferencias significativas en relación a la motivación intrínseca y a la motivación extrínseca, es decir, según los estudios cursados antes de iniciar la titulación ( $\mathrm{W}=13,52$; g.1. $=2$; g.l. $=179 ; \mathrm{p}<0,001$ y $\mathrm{F}=8,52$; g.l.=2; g.l.=987; $<<0,001$, respectivamente). Así, el alumnado que habían estudiado previamente un Ciclo Formativo de Grado Superior (CFGS) muestra una media más alta en la motivación intrínseca $(M=4,47)$. En el caso de la motivación extrínseca, la media más baja $(\mathrm{M}=2,37)$ corresponde a aquellos que han estudiado Bachillerato (véase la Tabla 3).

Según el momento en el que el alumnado decidió cursar el Grado, las medias relativas a la motivación intrínseca son significativamente diferentes $(\mathrm{W}=36,93$; g.l. $=5 ;$ g.l. $=265 ; \mathrm{p}<0,001$ ). Aquellos que han considerado estudiar Magisterio desde siempre presentan mayor media $(M=4,55)$ en motivación intrínseca. Sin embargo, aquellos que han tomado la decisión tras superar la Prueba de Acceso a la Universidad (PAU) alcanzan una puntuación menor $(\mathrm{M}=3,95)$ en este tipo de motivación.

Respecto a la motivación extrínseca, el alumnado que ha decidido cursar estos estudios al terminar el CFGS ( $\mathrm{M}=2,62)$ obtiene la media más elevada. Contrariamente, los que han indicado la opción desde siempre $(\mathrm{M}=2,36)$ o al terminar $\mathrm{ESO}(\mathrm{M}=2,37)$, las medias son más bajas. Constatándose que estas diferencias son estadísticamente significativas $(\mathrm{F}=3,72 ; \mathrm{g} .1 .=5 ; \mathrm{g} .1 .=979 ; \mathrm{p}=0,002)$.

En función del orden de elección de la titulación, el alumnado que ha optado por elegir esta carrera en primer lugar ha obtenido mayor media en la motivación intrínseca $(\mathrm{M}=4,40)$. Esta valoración disminuye a medida que se elige esta carrera en segundo, tercero, cuarto... lugar (Tabla 3). En este caso, dichas diferencias también resultan significativas estadísticamente $(\mathrm{W}=25,13 ; \mathrm{g} .1 .=3 ; \mathrm{g} .1 .=49 ; \mathrm{p}<0,001)$.

6 Los Ciclos Formativos de Grado Superior en España, forman parte de la educación superior y conducen a la obtención del título de Técnico Superior (Ministerio de Educación, Cultura y Deporte, 2016b).

7 La Educación Secundaria Obligatoria (ESO) es de carácter obligatorio y gratuito en España, consta de cuatro cursos académicos que se realizan ordinariamente entre los 12 y los 16 años de edad (Ministerio de Educación, Cultura y Deporte, 2016a).

8 La Prueba de Acceso a la Universidad (PAU) que se necesita hacer para acceder a los estudios universitarios en España, pretende garantizar que el alumnado tiene los conocimientos previos requeridos para cursar los estudios universitarios. Se compone de una fase general obligatoria y una fase específica voluntaria con la que se puede subir nota (Ministerio de Educación, Cultura y Deporte, 2016c). 
Revista de Humanidades, 38 (2019). p.37-64. ISSN 1130-5029

Tabla 3. Motivación intrínseca y extrínseca del alumnado de Magisterio, según las características académicas analizadas

\begin{tabular}{llcccccc}
\multicolumn{1}{l}{ Características académicas } & Motivación & & & \\
& Intrínseca & \multicolumn{3}{c}{ Extrínseca } \\
& $\mathrm{n}$ & Media & D.T. & $\mathrm{n}$ & Media & D.T. \\
\hline Estudios cursados & Bachillerato & 698 & 4,27 & 0,60 & 698 & 2,37 & 0,57 \\
antes de iniciar el & CFGS & 220 & 4,47 & 0,46 & 220 & 2,53 & 0,59 \\
primer curso del Grado & Otros estudios universitarios & 72 & 4,31 & 0,65 & 72 & 2,54 & 0,63 \\
\hline & Desde siempre & 421 & 4,55 & 0,43 & 421 & 2,36 & 0,60 \\
& Al terminar Bachillerato & 210 & 4,06 & 0,59 & 210 & 2,42 & 0,54 \\
Momento de elección & Al terminar CFGS & 99 & 4,33 & 0,53 & 99 & 2,62 & 0,54 \\
para realizar el Grado & Al terminar ESO & 86 & 4,24 & 0,53 & 86 & 2,37 & 0,53 \\
& Al terminar la PAU & 87 & 3,95 & 0,58 & 87 & 2,49 & 0,62 \\
& Otro & 82 & 4,22 & 0,78 & 82 & 2,38 & 0,61 \\
\hline \multirow{4}{*}{$\begin{array}{l}\text { Opción de elección de } \\
\text { la titulación }\end{array}$} & Primera & 779 & 4,40 & 0,52 & 779 & 2,41 & 0,59 \\
& Segunda & 168 & 4,09 & 0,61 & 168 & 2,44 & 0,55 \\
& Tercera & 22 & 3,68 & 0,60 & 22 & 2,37 & 0,66 \\
& Cuarta o más & 20 & 3,57 & 0,97 & 20 & 2,34 & 0,60 \\
\hline
\end{tabular}

Fuente. Elaboración propia.

\subsection{Características familiares}

Referente a las características familiares, analizamos las siguientes variables: a) estudios cursados por los padres (ninguno, leer y escribir, primarios, secundarios, superiores), b) nivel económico familiar (muy alto, alto, medio, bajo, muy bajo), c) trabajo remunerado del padre y de la madre (con las opciones: sí, no, realiza las labores del hogar) y d) la vinculación de dicha profesión con el ámbito de la enseñanza (sí, no), en el caso del padre y de la madre.

Los resultados de las medias de la motivación extrínseca son significativamente diferentes solo con respecto al nivel económico familiar $(\mathrm{F}=2,76$; g.1.=4; g.1.=953; $\mathrm{p}=0,027)^{9}$. Podemos observar en la Tabla 4 que la media más alta corresponde al alumnado con un nivel económico familiar alto $(\mathrm{M}=2,58)$. En relación a la motivación intrínseca no resulta estadísticamente significativa $(\mathrm{F}=0,44 ;$ g.l. $=4$; g.l. $=953 ; \mathrm{p}=0,779)$.

9 En las demás variables se ha obtenido un p-valor asociado al valor numérico del estadístico de la prueba mayor a 0,05 por lo que no se establecen diferencias significativas. Se obtienen para la motivación intrínseca y extrínseca respectivamente: estudios del padre $(\mathrm{F}=1,64$; g.l. $=4$; g.l. $=971$; $\mathrm{p}=0,161$ y $\mathrm{F}=0,24 ;$ g.l. $=4$; g.l. $=971 ; \mathrm{p}=0,917)$, estudios de la madre $(\mathrm{F}=1,34 ;$ g.l. $=4 ;$ g.l. $=975 ; \mathrm{p}=0,253$ y $\mathrm{F}=0,67 ;$ g.l. $=4 ;$ g.l. $=975 ; \mathrm{p}=0,613)$, trabajo remunerado del padre $(\mathrm{F}=2,00 ; \mathrm{g} .1 .=2 ; \mathrm{g} .1 .=966 ; \mathrm{p}=0,136$ y $\mathrm{W}=3,10 ;$ g.l. $=2 ;$ g.l. $=31 ; \mathrm{p}=0,059)$, trabajo remunerado de la madre $(\mathrm{F}=1,04 ;$ g.l. $=2 ; \mathrm{g} .1 .=975 ; \mathrm{p}=0,353$ y $\mathrm{F}=1,38 ;$ g.l. $=2$; g.l. $=975 ; \mathrm{p}=0,252)$, vinculación del trabajo del padre con la enseñanza $(\mathrm{t}=-0,34$; g.l.=769; $\mathrm{p}=0,737$ y $\mathrm{t}=1,82 ; \mathrm{g} .1 .=74 ; \mathrm{p}=0,072)$, vinculación del trabajo de la madre con la enseñanza $(\mathrm{t}=-0,49 ;$ g.l. $=459 ; \mathrm{p}=0,626$ y $\mathrm{t}=0,95 ;$ g.l. $=459 ; \mathrm{p}=0,345)$. 
Motivación de maestros de infantil y primaria... - V. Llorent-Bedmar y otros

Tabla 4. Motivación intrínseca y extrínseca del alumnado de Magisterio, según las características familiares analizadas

\begin{tabular}{|c|c|c|c|c|c|c|c|}
\hline \multirow[t]{3}{*}{ Características familiares } & \multicolumn{7}{|c|}{ Motivación } \\
\hline & \multirow[b]{2}{*}{$\mathrm{n}$} & \multicolumn{2}{|c|}{ Intrínseca } & \multicolumn{3}{|c|}{ Extrínseca } & \\
\hline & & Media & D.T. & $\mathrm{n}$ & Media & D.T. & \\
\hline \multirow{5}{*}{ Nivel económico familiar } & Muy alto & 2 & 4,50 & 0,47 & 2 & 2,54 & 0,38 \\
\hline & Alto & 46 & 4,29 & 0,68 & 46 & 2,58 & 0,66 \\
\hline & Medio & 736 & 4,33 & 0,58 & 736 & 2,42 & 0,59 \\
\hline & Bajo & 154 & 4,27 & 0,57 & 154 & 2,26 & 0,54 \\
\hline & Muy bajo & 20 & 4,23 & 0,55 & 20 & 2,51 & 0,42 \\
\hline \multirow{5}{*}{ Estudios del padre } & Ninguno & 22 & 4,17 & 0,57 & 22 & 2,53 & 0,58 \\
\hline & Leer y escribir & 31 & 4,42 & 0,52 & 31 & 2,39 & 0,66 \\
\hline & Primarios & 334 & 4,36 & 0,54 & 334 & 2,41 & 0,57 \\
\hline & Secundarios & 347 & 4,27 & 0,61 & 347 & 2,42 & 0,59 \\
\hline & Superiores & 242 & 4,31 & 0,61 & 242 & 2,41 & 0,59 \\
\hline \multirow{5}{*}{ Estudios de la madre } & Ninguno & 20 & 4,18 & 0,56 & 20 & 2,55 & 0,60 \\
\hline & Leer y escribir & 31 & 4,30 & 0,58 & 31 & 2,31 & 0,66 \\
\hline & Primarios & 315 & 4,37 & 0,55 & 315 & 2,43 & 0,59 \\
\hline & Secundarios & 358 & 4,30 & 0,57 & 358 & 2,40 & 0,57 \\
\hline & Superiores & 256 & 4,27 & 0,63 & 256 & 2,43 & 0,59 \\
\hline \multirow{3}{*}{$\begin{array}{l}\text { Trabajo remunerado del } \\
\text { padre }\end{array}$} & Sí & 776 & 4,32 & 0,58 & 776 & 2,39 & 0,57 \\
\hline & No & 180 & 4,28 & 0,59 & 180 & 2,50 & 0,59 \\
\hline & $\begin{array}{l}\text { No, realiza las } \\
\text { labores del } \\
\text { hogar }\end{array}$ & 13 & 4,62 & 0,52 & 13 & 2,69 & 0,87 \\
\hline \multirow{3}{*}{$\begin{array}{l}\text { Trabajo remunerado de la } \\
\text { madre }\end{array}$} & Sí & 457 & 4,31 & 0,59 & 457 & 2,44 & 0,59 \\
\hline & No & 142 & 4,26 & 0,59 & 142 & 2,46 & 0,56 \\
\hline & $\begin{array}{l}\text { No, realiza las } \\
\text { labores del } \\
\text { hogar }\end{array}$ & 379 & 4,34 & 0,55 & 379 & 2,39 & 0,57 \\
\hline \multirow{2}{*}{$\begin{array}{l}\text { Vinculación del trabajo del } \\
\text { padre con la enseñanza }\end{array}$} & Sí & 68 & 4,29 & 0,57 & 68 & 2,54 & 0,74 \\
\hline & No & 703 & 4,32 & 0,58 & 703 & 2,37 & 0,55 \\
\hline \multirow{2}{*}{$\begin{array}{l}\text { Vinculación del trabajo de la } \\
\text { madre con la enseñanza }\end{array}$} & Sí & 84 & 4,28 & 0,61 & 84 & 2,49 & 0,63 \\
\hline & No & 377 & 4,31 & 0,59 & 377 & 2,43 & 0,58 \\
\hline
\end{tabular}

Fuente. Elaboración propia.

\subsection{Características socio-económicas}

En cuanto a las características socio-económicas del alumnado hemos hallado diferencias significativas en: a) la realización de labores profesionales remuneradas 
Revista de Humanidades, 38 (2019). p.37-64. ISSN 1130-5029

y b) realización de actividades voluntarias en alguna organización. Los datos resultantes reflejan que aquellos que tienen un trabajo remunerado muestran medias de motivación intrínseca mayor $(\mathrm{M}=4,44)$ que aquellos que no disponen de empleo $(\mathrm{M}=4,30)$, siendo dicha diferencia significativa $(\mathrm{t}=2,31 ; \mathrm{g} .1 .=975 ; \mathrm{p}=0,021)$. En el caso de la motivación extrínseca, la media es mayor entre los que han señalado no tener trabajo remunerado; sin embargo, en este caso no se obtiene diferencia significativa $(\mathrm{t}=-0,43 ; \mathrm{g} .1 .=121 ; \mathrm{p}=0,670)$.

En el caso del voluntariado, la media de la motivación intrínseca es significativamente mayor para el alumnado que colabora como voluntario en alguna organización que la de aquellos que no participan en este tipo de actividades $(\mathrm{t}=2,88$; g.l.=962; p=0,004). En cambio, los universitarios que sí eran voluntarios alcanzan una media significativamente menor en motivación extrínseca $(\mathrm{t}=-2,49$; g.1. $=962$; $\mathrm{p}=0,013$ ), véase la Tabla 5 .

Tabla 5. Motivación intrínseca y extrínseca del alumnado de Magisterio, según las características socio-económicas analizadas

\begin{tabular}{lcccccccc}
$\begin{array}{l}\text { Características socio-económicas } \\
\text { del alumnado }\end{array}$ & \multicolumn{3}{c}{ Motivación } \\
\hline & \multicolumn{1}{c}{ Intrínseca } & \multicolumn{3}{c}{ Extrínseca } \\
\hline & $\mathrm{n}$ & Media & D.T. & $\mathrm{n}$ & Media & D.T. \\
\hline \multirow{2}{*}{ Trabajo remunerado } & Sí & 104 & 4,44 & 0,56 & 104 & 2,39 & 0,67 \\
& No & 873 & 4,30 & 0,58 & 873 & 2,42 & 0,57 \\
\hline \multirow{2}{*}{ Voluntario de alguna organización } & Sí & 134 & 4,45 & 0,55 & 134 & 2,30 & 0,64 \\
& No & 830 & 4,31 & 0,59 & 830 & 2,41 & 0,57 \\
\hline
\end{tabular}

Fuente. Elaboración propia.

Durante el desarrollo de las entrevistas, además de los factores estudiados, profundizamos sobre las razones por las cuales los universitarios de ambos Grados se sienten más motivados. Los participantes de Educación Infantil, expresan mayoritariamente sentirse más motivados debido a que los estudios les permiten mejorar en su crecimiento personal $(30,8 \%)$ y a la realización de Prácticas de Enseñanza (26,9\%). En el caso de los entrevistados de Educación Primaria, en mayor proporción indican que el aspecto por el que se sienten más motivados durante los estudios se debe a que pueden tener cercanía con los niños (35,7\%); también señalan como razones que les motivan la adquisición de nuevos aprendizajes $(21,4 \%)$ y el desarrollo de Prácticas de Enseñanza (21,4\%).

Asimismo, durante las entrevistas cuestionamos al alumnado los aspectos que durante los estudios los podían animar para el desarrollo de su titulación. En el Grado de Educación Infantil señalan desarrollar contenidos más actuales y prácticos $(33,3 \%)$, ampliar el periodo de prácticas externas $(31,2 \%)$, mejorar la organización 
del plan de estudios $(8,3 \%)$, mayor coordinación entre los docentes $(8,3 \%)$, mayor nota de acceso $(6,3 \%)$ y cambios en el proceso de evaluación $(4,2 \%)$. En el Grado de Educación Primaria indican ampliar el periodo de prácticas externas, $(22 \%)$, establecer contenidos más actuales y prácticos $(22 \%)$, optimizar la organización del plan de estudios $(22 \%)$, facilitar la participación de los discentes $(9,8 \%)$, cambiar el proceso de evaluación $(7,3 \%)$ y mejorar la coordinación entre los docentes universitarios $(7,3 \%)$.

Finalmente, respecto a los rasgos de los docentes universitarios para el buen desarrollo de los estudios, el alumnado de los Grados de Educación Infantil y Primaria fundamentalmente expresa durante las entrevistas que tengan cualidades para comunicar $(40,7 \%$ y $37,9 \%$, respectivamente) y sean cercanos $(24,1 \%$ en ambos Grados).

\section{DISGUSIÓN Y GONGLUSIONES}

Hemos podido comprobar que determinados factores y características del alumnado universitario de Magisterio se relacionan con su motivación intrínseca y extrínseca. Este aspecto resulta relevante para diseñar una adecuada formación inicial, ya que, como diferentes autores indican, nuestra acción y las estrategias de aprendizaje utilizadas son diferentes en función del tipo de motivación (entre otros, Henaín, 2015; Ahn y Shin, 2014; y Rinaudo et al., 2003).

Además, deseamos reincidir que la motivación va cambiando según los momentos y circunstancias. Debemos tener presente que la motivación no permanece estable a lo largo de la vida, aumenta o disminuye en función de determinados factores y nuevas experiencias vividas. La motivación implica un dinamismo funcional, debido a la necesaria adaptación a las circunstancias que ocurren en el entorno del alumnado (Palmero, 2005). De aquí la importancia del objetivo general planteado, resulta necesaria la concienciación hacia la mejora de la motivación intrínseca del alumnado de Magisterio, ya que desde el ámbito universitario, concretamente los docentes que imparten en las titulaciones de Magisterio debemos ser conscientes de ello durante la formación en el Grado y establecer las medidas oportunas.

Partiendo de esta premisa, los resultados obtenidos permiten establecer estrategias que mejoren la motivación intrínseca del alumnado universitario de Magisterio:

1. Consideramos importante definir la motivación intrínseca y extrínseca, a lo que nos referimos en el primero de los objetivos específicos. Los resultados de esta investigación ponen de manifiesto que, a medida que aumenta la motivación intrínseca, se produce una disminución en la motivación extrínseca, según los ítems estudiados. Este aspecto es de gran importancia para el profesorado universitario que imparte docencia en los Grados de 
Revista de Humanidades, 38 (2019). p.37-64. ISSN 1130-5029

Educación Infantil y Educación Primaria, pues al establecer estrategias que desarrollen la motivación extrínseca, pueden obtenerse resultados a corto plazo (por ejemplo, la adquisición memorística de conocimiento para un examen), pero pueden ir en detrimento de la motivación intrínseca (la adquisición de un conocimiento significativo para ponerlo en práctica durante su futuro desempeño profesional).

En este sentido, Henaín (2015) y Rinaudo et al. (2003) afirman que el tipo de motivación que tiene el alumnado incide en su estilo de aprendizaje. Por tanto, el desarrollo de la motivación intrínseca en el aprendizaje es de gran importancia. De acuerdo con De Cooman et al. (2007), los docentes recién titulados que ejercen la profesión consideran los aspectos de carácter intrínseco, altruista e interpersonal como relevantes para su desempeño profesional. Afirmaciones que apoyan la importancia de los resultados obtenidos.

2. Respecto al análisis de los factores de índole académica, familiar y socioeconómica (segundo objetivo específico) y su relación con el nivel y tipo de motivación (tercer objetivo específico), los resultados permiten concluir y establecer las siguientes propuestas:

A. En relación a las características académicas, hallamos que el alumnado que tenía pensado estudiar las titulaciones de Educación Infantil y Educación Primaria desde pequeños ("desde siempre") tiene mayor motivación intrínseca que aquellos que lo decidieron al terminar la PAU.

Respecto al orden de elección para el acceso a la universidad, el alumnado que eligió la titulación de Magisterio como primera opción manifestó mayor nivel de motivación intrínseca.

Estos resultados ponen de relieve la necesidad de ofrecer programas adecuados de orientación académica y profesional dentro del sistema escolar de un país (Meijers et al., 2013). La relevancia de una orientación vocacional dirigida a todo el alumnado antes de cursar los estudios superiores, la entendemos como un proceso longitudinal que se desarrolle desde la infancia hasta la edad adulta y que esté determinada por factores personales y contextuales y no meramente de carácter profesional (Araújo y Taveira, 2009). En la formación vocacional, previa a la elección de la titulación, resulta necesaria una educación personalizada que permita el desarrollo integral de la persona. En España, la Ley Orgánica 2/2006, de 3 de mayo, de Educación (LOE), sin modificación en la actual Ley Orgánica 8/2013, de 9 de diciembre, para la Mejora de la Calidad Educativa (LOMCE) establece lo siguiente: “(...) f) La orientación educativa y profesional de los estudiantes, como medio necesario para el logro de una formación personalizada, que propicie una educación integral en conocimientos, destrezas y valores" (LOE, 2006, artículo 1). 
Continuando con las características académicas, los resultados también evidencian que el alumnado que cursó previamente un CFGS presenta una motivación intrínseca mayor que los que proceden de Bachillerato.

En este sentido, manifestamos la necesidad de llevar a cabo un replanteamiento del sistema de acceso a las titulaciones de Magisterio en España. Han sido muchos los debates acerca de la necesidad de aumentar la nota de corte en dichas titulaciones, en efecto, en los últimos años hemos podido constatar que en algunas facultades de Ciencias de la Educación se ha incrementado la nota de acceso en los Grados de Educación Infantil y Primaria. Como ejemplo, el caso de la Universidad de Sevilla, en la titulación de Infantil la nota de corte en 2010 es 8,600 y en 2017 es 9,333; para el Grado de Primaria en 2010 es 7,271 y en 2017 es 8,580 (Distrito Único Andaluz, 2017).

Si bien, un procedimiento que endurece los requisitos de acceso a la titulación puede estar vinculado a la mejora del prestigio social de dicha profesión, sin embargo, debemos tener presente que el incremento de la nota de corte impediría cursar los estudios a discentes que pueden ser buenos docentes motivados intrínsecamente.

Asimismo, debemos tener en cuenta el dato anteriormente indicado, referido a que el alumnado que procede del CFGS muestra mayor motivación intrínseca. En este sentido, según manifestaron la mayoría de los encuestados del Grado de Educación Infantil que procedían de CFGS, la especialidad que habían cursado previamente tenía clara relación con la temática de la titulación, como por ejemplo Técnico Superior en Educación Infantil. Así, podemos percibir que cuando el alumnado aprende conocimientos más vinculados a la profesión de Magisterio (como en CFGS) mejora su ánimo por estos estudios.

En esta misma línea, diferentes autores abogan por modificar el acceso a estas titulaciones, atendiendo a las habilidades y capacidades relacionadas con la docencia. Es decir, teniendo más en cuenta determinadas aptitudes necesarias para ser un buen maestro. Tal como defienden Zapico-Barbeito et al. (2017) "la necesidad de poseer ciertas características personales que no se tienen en cuenta o que no se precisan para obtener una nota alta en la selectividad" (p. 93).

B. Determinados aspectos de índole familiar también se relacionan con el tipo de motivación del alumnado, aunque han sido las menos relevantes. Así pues, los que presentan un nivel económico familiar bajo, manifiestan tener menor motivación extrínseca.

Figuera y Álvarez (2014) en este sentido, proponen "articular acciones de información orientadas a conocer la realidad socioeconómica de las familias y a la posibilidad de consecución de becas para lograr los objetivos personales formativos" (p. 39). 
Revista de Humanidades, 38 (2019). p.37-64. ISSN 1130-5029

No obstante, el análisis realizado sobre las características familiares del alumnado en relación a la motivación intrínseca no resulta estadísticamente significativo (véase Tabla 5). Esto nos resulta lógico, ya que las características familiares son un factor externo que pueden ejercer menor influencia en la motivación intrínseca que otros factores como el gusto por la enseñanza, el disfrute al trabajar con niños o la vocación por la profesión, entre otros.

Resaltamos este hecho para incidir en la importancia de la elección de la titulación de manera personal y razonada. De acuerdo con Haguinzaca (2017) la elección de la titulación, y consecuentemente la profesión, se debe realizar "con amor y así puedan ser más eficaces y eficientes en su área laboral o como estudiante" (p. 6). Por el contrario, la falta de decisión en la elección de la titulación por influencia de familiares o por el grupo de iguales "los lleva a una decisión incorrecta, logrando así una frustración en el estudiante" (p. 17). Además, indican Fernández-García et al. (2016) que "los padres, mantienen que la elección académica realizada es una responsabilidad de los hijos, es una decisión eminentemente individual que condicionará en buena medida su vida profesional futura" (p. 1120). Por consiguiente, volvemos a incidir en la importancia del desarrollo de programas de formación vocacional que favorezcan la toma de decisiones del alumnado de manera crítica.

C. Respecto a las características socio-económicas, el alumnado de los Grados de Educación Infantil y Primaria que desempeñan un trabajo remunerado y/o aquellos que participan en actividades de voluntariado, presentan mayor motivación intrínseca que el resto.

Así pues, teniendo en cuenta que la motivación varía según los estímulos recibidos, proponemos fomentar unas condiciones externas que puedan propiciar la mejora de la motivación intrínseca, tales como el desempeño de labores de voluntariado en el alumnado. En este sentido, el Instituto de Estudios Laborales de ESADE afirma que "el 92\% de los trabajadores que participan en actividades de voluntariado mejoran su rendimiento (...) Las capacidades que más aumentan son las de trabajar en equipo y comprender la realidad social (...)" (ESADE, 2014).

En definitiva, dada la relevancia de la figura del docente en la motivación del alumnado (Espinar y Ortega, 2015), son convenientes investigaciones que estudien la motivación de futuros maestros y de los factores que en esta pueden repercutir. El futuro maestro debe estar suficientemente motivado para ejercer su labor docente, favoreciendo en su alumnado el gusto por aprender. Solo si es así puede aplicar estrategias que motiven (González-Peiteado, 2013). En consecuencia, debemos fomentar el desarrollo de la motivación en la formación inicial del maestro, especialmente la de carácter intrínseco. 
De acuerdo con Herraiz (2015) "los contextos de formación son complejos y variables" (p. 206), son diversas las formas en las que se puede producir tanto el proceso de aprendizaje inicial del docente como la forma en la que este ejerce la profesión. Por tanto, los hallazgos de esta investigación son de utilidad, por un lado, para favorecer programas adecuados de orientación vocacional y, por otro, para mejorar los planes de estudio de la formación inicial de los futuros maestros y concienciar al profesorado responsable de dicha formación.

Así, consideramos que los objetivos propuestos en este artículo se han cumplido. A partir de los resultados obtenidos es conveniente plantear nuevas líneas de investigación, consideramos necesario investigar la relación de la motivación con otros factores, tales como el nivel de autoeficacia del alumnado, el estrés o la percepción que tiene del docente, entre otros. Asimismo, creemos de gran importancia el desarrollo de proyectos de innovación docente para la mejora de la motivación intrínseca en el alumnado que cursa Magisterio, de esta forma pueden establecerse actuaciones concretas en los centros y facultades donde se imparten los Grados de Educación Infantil y Educación Primaria.

\section{REFERENCIAS BIBLIOGRÁFICAS}

Ahn, Doehee y Shin, Min (2014). Factors Influencing Competence: On Academic Motivation and Learning Strategies of Gifted and Non-gifted Students. Journal of Gifted/Talented Education, 24(1), 1-16. doi: 10.9722/ JGTE.2014.24.1.1

Albert, Ma. José (2007). La Investigación Educativa. Claves teóricas. Madrid: Mc Graw Hill.

Aliakbari, Mohammad y Hemmatizad, Marzieh (2015). On students' de-motivation, gender, major, and educational level in iranian EFL context. English Language Teaching, 8(4), 106-116. doi: http://dx.doi.org/10.5539/elt.v8n4p106

Araújo, Alexandra y Taveira, Maria do Céu (2009). Estudio del desarrollo de la orientación vocacional en la infancia desde la perspectiva evolutivo-contextual. European Journal of Education and Psychology, 2(1), 49-67. doi:http://dx.doi. org/10.1989/ejep.v2i1.19

Arias, Bentio (2008). Desarrollo de un ejemplo de Análisis Factorial Confirmatorio con Lisrel, Amos y Sas. En Miguel Ángel Verdugo, Manuela Crespo, Marta Badía y Benito Arias (Coords.), Simposio Científico SAID, 2008. Metodología en la investigación sobre discapacidad. Introducción al uso de las ecuaciones estructurales. Recuperado de http://sid.usal.es/idocs/F8/FDO20749/said_2008. pdf

Bailey, Thomas Hamilton y Phillips, Lisa J. (2016). The influence of motivation and adaptation on students' subjective well-being, meaning in life and academic performance. Higher Education Research \& Development, 35(2,3), 201-216. doi:http://dx.doi.org/10.1080/07294360.2015.1087474 
Revista de Humanidades, 38 (2019). p.37-64. ISSN 1130-5029

Bertomeu, Felipe J., Canet, Glòria, Gil, Verónica y Jarabo, Juan A. (2007). Las motivaciones hacia los estudios de magisterio. Recuperado de http://repositori. uji.es/xmlui/bitstream/handle/10234/78666/forum_2006_32.pdf?sequence=1

Bhuiyan, Pritha, Supe, Avinash y Rege, Nirmala (2015). The Art of Teaching Medical Students ( $3^{\mathrm{a}}$ ed.). Haryana, India: Elsevier

Bisquerra, Rafael (2000). Métodos de investigación educativa. Guía práctica. Barcelona, España: Ceac educación

Boza, Ángel y Toscano, María de la O (2012). Motivos, actitudes y estrategias de aprendizaje: aprendizaje motivado en alumnos universitarios. Revista de currículum y formación del profesorado, 16(1), 125-142. Recuperado de http:// www.ugr.es/ recfpro/rev161ART8

Cabanach, Ramón G., Valle, Antonio, Rodríguez, Susana, Piñeiro, Isabel y González, Patricia (2010). Las creencias motivacionales como factor protector del estrés en estudiantes universitarios. European Journal of Education and Psychology, 3(1), 75-87. doi: 10.1989/ejep.v3i1.47

De Boer, Hester, Bosker, Roel, J. y Van der Werf, Margaretha P.C. (2010). Sustainability of teacher expectation bias effects on long-term student performance. Journal of Educational Psychology, 102(1). doi: http://dx.doi. org/10.1037/a0017289

De Cooman, Rein, De Gieter, Sara, Pepermans, Roland, Du Bois, Cindy, Caers, Ralf y Jegers, Marc (2007). Graduate teacher motivation for choosing a job in education. International Journal for Educational and Vocational Guidance, 7(2), 123-136. doi: 10.1007/s10775-007-9117-5

Del Siegle, Rubenstein, Lisa DaVia y Mitchell, Melissa S. (2014). Honors students' perceptions of their high school experiences: The influence of teachers on student motivation. Gifted child quarterly, 58(1), 31-50. Recuperado de http://gcq. sagepub.com $/$ content $/ 58 / 1 / 35$.short? $r s s=1 \&$ ssource $=\mathrm{mfr}$

Distrito Único Andaluz (2017). Notas de corte de años anteriores. Recuperado de http://www.juntadeandalucia.es/economiainnovacionyciencia/sguit/g_not_cor_ anteriores_top.php

Donche, Vicent, De Maeyer, Sven, Coertiens, Liesje, Van Daal, Tine y Van Petegem, Peter (2013). Differential use of learning strategies in first-year higher education: the impact of personality, academic motivation, and teaching strategies. British Journal of Educational Psychology, 83(2), 238-251. doi: 10.1111/bjep.12016

Dörnyei, Zoltán y Ushioda, Ema (2013). Teaching and Research Motivation. New York: Routledge

Dryer, Rachel, Henning, Marcus A., Tyson, Graham A., y Shaw, Rosemary (2016). Academic Achievement Performance of University Students with Disability: Exploring the Influence of Non-academic Factors. International Journal of Disability, Development and Education, 1-12. doi: 10.1080/1034912X.2015.1130217 
Motivación de maestros de infantil y primaria... - V. Llorent-Bedmar y otros

Elias, Marina y Sánchez-Gelabert, Albert (2014). Relación entre actitudes y acciones de aprendizaje de los estudiantes universitarios. Revista de Estudios e Investigación en Piscología y Educación, 1(1), 3-14. doi: : 10.17979/reipe.2014.1.1.17

ESADE (2014). El 95\% de los trabajadores que participan en actividades de voluntariado mejoran su rendimiento, según un estudio de ESADE. Recuperado de http://www. esade.edu/es/news/el-92-de-los-trabajadores-que-participan-en-actividades-devoluntariado-mejoran-su-rendimiento-segun-un-estudio-de-esade/1687-303022

Espinar, Rocío y Ortega, José Luis (2015). Motivation: The road to successful learning. PROFILE Issues in Teachers' Professional Development, 17(2), 125-136. doi: http://dx.doi.org/10.15446/profile.v17n2.50563

Fernández-García, Carmen María, García-Pérez, Omar y Rodríguez Pérez, Sara (2016). Los padres y madres ante la toma de decisiones académicas de los adolescentes en la educación secundaria. Un estudio cualitativo. Revista Mexicana de Investigación Educativa, 21(71), 1111-1133. Recuperado de http://www.redalyc.org/articulo. oa? $\mathrm{id}=14047430006$

Field, Andy (2009). Discovering Statistics using SPSS (3 ${ }^{\text {a }}$ ed.). Londres: SAGE Publications.

Figuera, Pilar y Álvarez, Manuel (2014). La intervención orientadora y tutorial en la adaptación y persistencia del alumnado en la Universidad. Revista de Orientación Educacional, 28(54), 31-49. Recuperado de http://www.roe.cl/index.php/roe/issue/ view/8

García-Ramirez, José Miguel (2016). La motivación de logro mejora el rendimiento académico. ReiDoCrea, 5, 1-8. doi: 10.13140/RG.2.1.3945.4480

García, María Inés (2011). Estudio sobre las metas de elección de carrera en los estudiantes que ingresas a la Pontificia Universidad Católica Argentina. Recuperado de http:// www.uca.edu.ar/mailing/ingreso/2011-08-Estudio-sobre-las-metas-de-eleccionde-carrera-Lic-Ines-Garcia-Ripa.pdf

González-Peiteado, Margarita (2013). Los estilos de enseñanza y aprendizaje como soporte de la actividad docente, Journal of Learning Styles, 11(11), 51-70. Recuperado de http://www2.uned.es/revistaestilosdeaprendizaje/numero_11/ 1sr_11_abril_2013.pdf

Haguinzaca, Stalin Omar (2017). Plan de orientación vocacional y motivacional para estudiantes con problema de elección de carrera basado en la teoría psicológica humanista. Recuperado de http://repositorio.utmachala.edu.ec/handle/48000/10451

Herrero, Juan (2010). El Análisis Factorial Confirmatorio en el estudio de la Estructura y Estabilidad de los Instrumentos de Evaluación: Un ejemplo con el Cuestionario de Autoestima CA-14. Intervención Psicosocial, 19(3), 289-300. doi: 10.5093/ in2010v19n3a9

Henaín, Yamile (2015). Estudio del rendimiento académico de los estudiantes de Licenciatura en Nutrición de la Universidad Nacional del Litoral que comenzaron en el año 2008, y su relación con los factores motivacionales y las expectativas 
Revista de Humanidades, 38 (2019). p.37-64. ISSN 1130-5029

que tuvieron al ingreso en la carrera _ Santa Fe, año 2011. Tesis para la obtención del grado académico de Magíster en docencia universitaria. Universidad Nacional del Litoral, Facultad de Humanidades y Ciencias. Recuperado de http:// bibliotecavirtual.unl.edu.ar:8080/tesis/handle/11185/715

Herraiz, Fernando (2012). Universidad y la vida laboral en la Escuela de maestros y maestros noveles. Reflexionando en torno a identidades docentes y sus aprendizajes. Profesorado. Revista de currículum y formación del profesorado, 19(2), 203-214. Recuperado de http://www.ugr.es/ recfpro/rev192ART12.pdf

Hidi, Suzanne (2015). Revisiting the Role of Rewards in Motivation and Learning: Implications of Neuroscientific Research. Educational Psychology Review, 28(1), 61-93. doi: 10.1007/s10648-015-9307-5

Hopper, Daire, Coughlan, Joseph, y Mullen, Michael R. (2008). Structural Equation Modelling: Guidelines for Determining Model Fit. Electronic Journal of Business Research Methods, 6(1), 53-60. Recuperado de http://www.ssnpstudents.com/wp/ wp-content/uploads/2015/02/ejbrm-volume6-issue1-article183.pdf

Hu, Li-tze y Bentler, Peter M. (1999). Cutoff Criteria for Fit Indexes in Covariance Structure Analysis: Conventional Criteria Versus New Alternatives. Structural Equation Modeling, 6 (1), 1-55

Ilustrisimo, Ruby D. (2016). Exploring links between time perspective and academic motivation among filipino undergraduates. The Psychology of Asian Learners: A Festschrift in Honor of David Watkins: 289-300.Recuperado de http:// link.springer.com/chapter/10.1007\%2F978-981-287-576-1_18

Lei, Pui-Wa y Wu, Qiong (2007). Introduction to Structural Equation Modeling: Issues and Practical Considerations. Educational Measurement: Issues and Practice, 26, 33-43. doi: 10.1111/j.1745-3992.2007.00099.x

Ley Orgánica 8/2013, de 9 de diciembre, para la Mejora de la Calidad Educativa. BOE $\mathrm{n}^{\circ} 295(2013)$

Ley Orgánica 2/2006, de 3 de mayo, de Educación. BOE nº 106 (2006)

Liu, Woon Ch., Wang, Chee K.J., Kee, Ying H., Koh, Caroline, Lim, Boon S.C. y Chua, Lilian (2014). College Students' Motivation and Learning Strategies Profiles and Academic Achievement: A Self-Determination Theory Approach. Educational Psychology, 34(3), 338-353. doi: 10.1080/01443410.2013.785067

McMillan, James H. y Schumacher, Sally (2005). Investigación educativa (5 $5^{\mathrm{a}}$ ed.) Madrid, España: Pearson Educación

Meijers, Frans, Kuijpers, Marinka y Gundy, Chad (2013). The relationship between career competencies, career identity, motivation and quality of choice. International Journal for Educational and Vocational Guidance, 13(11), 4766. doi: 10.1007/s10775-012-9237-4

Ministerio de Educación, Cultura y Deporte (2016a). Sistema educativo. Recuperado de http://www.mecd.gob.es/educacion-mecd/areas-educacion/sistema-educativo.html 
Motivación de maestros de infantil y primaria... - V. Llorent-Bedmar y otros

Ministerio de Educación, Cultura y Deporte (2016b). El sistema educativo y la formación profesional. Recuperado de http://www.todofp.es/todofp/sobre-fp/informaciongeneral/sistema-educativo-fp.html

Ministerio de Educación, Cultura y Deporte (2016c). Universidades. Recuperado de http://www.mecd.gob.es/educacion-mecd/areas-educacion/universidades.html

Naranjo, María Luisa (2009). Motivación: perspectivas teóricas y algunas consideraciones de su importancia en el ámbito educativo. Revista Educación, 33(2), 153-170

Niehaus, Kate, Moritz, Kathleen y Adelson, Jill L. (2012). Self-Efficacy, Intrinsic Motivation, and Academic Outcomes Among Latino Middle School Students Participating in an After-School Program, Hispanic Journal of Behavioral Sciences, 34(1), 118-136. doi: 10.1177/0739986311424275

Padilla, María Teresa (2002). Técnicas e instrumentos para el diagnóstico y la evaluación educativa. Madrid, España: Editorial CCS.

Palmero, Francesc (2005). Motivación: conducta y proceso. Revista Electrónica de Motivación y Emoción, 8(20-21). Recuperado de http://reme.uji.es/articulos/ numero20/1-palmero/texto.html

Pan, Irene, Regueiro, Bibiana, Ponte, Beatriz, Rodríguez, Susana, Piñeiro, Isabel y Valle, Antonio (2013). Motivación, implicación en los deberes escolares y rendimiento académico. Aula Abierta, 41(3), 13-22. Recuperado de https://dialnet.unirioja.es/ servlet/articulo? $\operatorname{codigo}=4401103$

Pereira, Zulay (2011). Los diseños de método mixto en la investigación en educación: una experiencia concreta. Revista Electrónica Educare, 15(1), 15-29. Recuperado de http://www.redalyc.org/articulo.oa?id=194118804003

Pérez, Ramón, Galán, Arturo y Quintanal, José (2012). Métodos y diseños de investigación en educación. Madrid, España: Universidad Nacional de Educación a Distancia.

Presentación, María Jesús, Pinto, Vicente, Mercader, Jessica, Colomer, Carla, Siegenthaler, Rebeca y Miranda, Ana (2015). Motivación y estilo atribucional sobre el rendimiento académico en Educación Infantil. International Journal of Developmental and Educational Psychology. Revista Infad de Psicología, 1(1), 101-110. doi: http://dx.doi.org/10.17060/ijodaep.2015.n1.v1.52

Real Decreto 412/2014, de 6 de junio, por el que se establece la normativa básica de los procedimientos de admisión a las enseñanzas universitarias oficiales de Grado, BOE $n^{\circ} 138$ (2014)

Reyes, Nancy (2015). Motivación del estudiante y los entornos virtuales de aprendizaje. Recuperado de http://reposital.cuaed.unam.mx:8080/jspui/handle/123456789/3812

Rinaudo, María Cristina, Chiecher, Analía y Donolo, Danilo (2003). Motivación y uso de estrategias en estudiantes universitarios. Su evaluación a partir del Motivated Strategies Learning Questionnaire. Anales de Psicología, 19(1), 107-119

Rodríguez, Gustavo (2009). Motivación, estrategias de aprendizaje y rendimiento académico en estudiantes de E.S.O. (Tesis doctoral). Universidad de La Coruña, Facultad de Ciencias de la Educación, Departamento de Psicología Evolutiva y de la Educación. 
Revista de Humanidades, 38 (2019). p.37-64. ISSN 1130-5029

Rosales, Carlos (2014). Competencias específicas curriculares que ha de adquirir el estudiante del Título de Grado de Maestro. Profesorado. Revista de currículum y formación del profesorado, 17(3), 73-90. Recuperado de http://www.ugr. es/ recfpro/rev173ART4.pdf

Rosseel, Yves (2015). The lavaan tutorial. Recuperado de http://lavaan.ugent.be/tutorial/ tutorial.pdf

Ruiz, Miguel A., Pardo, Antonio y San Martín, Rafael (2010). Modelos de Ecuaciones Estructurales, Papeles del psicólogo, 31(1), 34-45. Recuperado de https://dialnet. unirioja.es/servlet/articulo?codigo $=3150815$

Sañudo, Lya E. (2006). La ética en la investigación educativa, Hallazgos, 3(6), 83-98. doi: https://doi.org/10.15332/s1794-3841.2006.0006.05

Sarsenbaykyzy, Guldana, Meterbayevna, Kulbarshyn, Ametbekovich, Azamat y Eletaevna, Gulnar (2016). Motives for Choosing the Profession of a Social Care Teacher in the Universities of Kazakhstan, International Review of Management and Marketing, 6(3S), 133-139. Recuperado de http://www.econjournals.com/ index.php/irmm/article/view/2202

Siwek, Zuzanna, Oleszkowicz, Anna y Słowińska, Aleksandra (2016).Values Realized in Personal Strivings and Motivation, and Meaning in Life in Polish University Students. Journal of Happiness Studies, 1-25. doi: 10.1007/ s10902-016-9737-x

Valle, Antonio, Regueiro, Bibiana, Rodríguez, Susana, Piñeiro, Isabel, Freire, Carlos, Ferradás, Mar y Suárez, Natalia (2015). Perfiles motivacionales como combinación de expectativas de autoeficacia y metas académicas en estudiantes universitarios. European Journal of Education and Psychology, 8(1), 1-8. Recuperado de http:// www.sciencedirect.com/science/article/pii/S1888899215000082

Valles, Miguel S. (2014). Entrevistas cualitativas (2ª ed.). Madrid, España: Centro de Investigaciones Sociológicas

Vivanco, Manuel (2005). Muestreo Estadístico. Diseño y Aplicaciones. Santiago de Chile, Chile: Editorial Universitaria

Zapico-Barbeito, María-Helena, Martínez Piñeiro, Esther y Montero, María Lourdes (2017). Demandas sociales y formación inicial del profesorado ¿un callejón sin salida? Profesorado. Revista de currículum y formación del profesorado, 21(1), 80-102. Recuperado de https://recyt.fecyt.es/index.php/profesorado/article/ view/58051 